\section{$\underset{\substack{\text { hommes } \\ \text { \& migrations }}}{ }$}

\section{Hommes \& migrations}

Revue française de référence sur les dynamiques

migratoires

$1284 \mid 2010$

Migrations et environnement

\title{
Combattre "nos bagnes et nos ravages"
}

\section{Mustapha Harzoune}

\section{(QpenEdition \\ Journals}

\section{Édition électronique}

URL : http://journals.openedition.org/hommesmigrations/1269

DOI : 10.4000/hommesmigrations.1269

ISSN : 2262-3353

\section{Éditeur}

Musée national de l'histoire de l'immigration

\section{Édition imprimée}

Date de publication : 1 mars 2010

Pagination : 180-188

ISSN : 1142-852X

Référence électronique

Mustapha Harzoune, "Combattre "nos bagnes et nos ravages" », Hommes \& migrations [En ligne],

1284 | 2010, mis en ligne le 29 mai 2013, consulté le 22 septembre 2020. URL : http://

journals.openedition.org/hommesmigrations/1269; DOl : https://doi.org/10.4000/

hommesmigrations.1269

Ce document a été généré automatiquement le 22 septembre 2020.

Tous droits réservés 


\title{
Combattre "nos bagnes et nos ravages"
}

\author{
Mustapha Harzoune
}

Branle-bas de combat dans la presse autour d'Albert Camus, disparu le 4 janvier 1960, il y a cinquante ans tout juste. Une mobilisation médiatique grossie d'une nouvelle idée présidentielle : transférer celui qui repose sous le soleil de Lourmarin dans la crypte du Panthéon. Camus, d'origine espagnole par sa mère, fils de colon dans une Algérie française aux allures d'apartheid, Camus, "le fils du pauvre", modeste gamin de Belcourt, l'Algérien souffreteux et anxieux, placé entre la misère et le soleil... celui-là même qui subit les quolibets de l'intelligentsia germanopratine dont l'écho raisonne encore aux oreilles des moins oublieux. "Qui a tué Camus?" demande Olivier Mony dans Le Figaro du 7 janvier 2010. "Personne, bien sûr. Personne, sinon le chagrin, l'insondable tristesse de celui qui eut raison si tôt qu'il crut s'être enfoncé dans l'erreur, de celui dont le doute accompagna chaque pas, celui pour qui le succès ne fut jamais un baume mais presque une souffrance. Celui qui, quelques jours après sa plus grande gloire, de retour de Stockholm, écrit dans son journal de bord: '29 décembre (1957), 15 heures, nouvelle crise panique. [...] Pendant quelques minutes, sensation de folie totale. Ensuite, épuisements et tremblements. Calmants. [...] Nuit du 29 au 30 : interminables angoisses. [...] 1er janvier, anxiété redoublée.' Celui-là était inconsolable."

2 Si la France célèbre Camus, en Algérie, la presse est plus divisée et, ici ou là, percent quelques perfidies d'un autre âge à l'endroit d'un homme dont le seul tort aurait peutêtre été de refuser l'indépendance d'une Algérie dominée par le FLN. C'est ce que rappelle dans El Watan du 25 décembre 2009, José Lenzini : Camus "ne croyait pas à la possibilité des différentes communautés de se retrouver dans l'harmonie d'une indépendance, qui lui paraissait vouée à de grosses contradictions du fait de son 'usurpation' par le FLN." Se trouver dans le camp de la justice ne dédouane pas de ses responsabilités. Comme l'écrivait le 4 janvier, Maurice Ulrich dans L'Humanité: "Sa révolte n'est pas contre la condition humaine, mais contre ce qui asservit l'homme. "Nous portons tous en nous nos bagnes et nos ravages. Mais notre tâche n'est pas de les déchaîner à travers le monde. Elle est de les combattre en nous-mêmes et dans les autres' (L'Homme révolté)." 


\section{Réfléchir comme Camus}

Alors! Camus en Algérie? Catherine Gouëset, sur le site de L'Express constate que “l'anniversaire de la mort d'Albert Camus est assez peu présent, ce 4 janvier dans la presse algérienne de langue française, reflétant le malaise, voire le rejet, suscité par l'auteur de L'Étranger auprès des Algériens." Il faut lire ce Quotidien d'Oran du 11 janvier où avec "Camus, une perte algérienne", Akram Belkaïd fournit la plus synthétique, visionnaire et stimulante contribution.

4 Synthétique : “C'est peut-être en Algérie où l'on a le plus parlé de Camus ces dernières années et où l'on n'a pas attendu la date du 4 janvier pour se souvenir de lui. [...] Il sera difficile de réconcilier les deux camps: ceux qui, comme jadis Kateb Yacine, lui reprochent d'avoir nié dans ses écrits littéraires les indigènes, et ceux qui retiennent à la fois sa fidélité à sa terre natale, ses engagements intellectuels et la force de ses écrits."

5 Visionnaire: “Ce qui m'importe, ce n'est pas de savoir si Camus doit être considéré comme algérien ou non. Tôt ou tard, il y aura une rue dans le pays qui finira par porter son nom, peut-être même un lycée, voire une bibliothèque ou un centre culturel. Il y aura certainement des protestations de la part de certains membres de la famille révolutionnaire héréditaire, mais cela se tassera. Les Algériens savent aujourd'hui qu'apprécier Camus et lui rendre hommage (sans verser dans l'hagiographie intéressée, comme le font certains écrivains algériens installés en France) ne signifie pas une adhésion au hizb França, ni une remise en cause de l'indépendance. Après tout, Camus n'était peut-être qu'un Algérien qui ne pensait pas comme nombre de ses compatriotes..."

6 Stimulante: "En somme, ce que nous avons perdu avec Camus, c'est le refus du manichéisme, le refus d'accepter les vérités assénées, les principes érigés en dogme, les certitudes humaines transformées en lois suprêmes. C'est une banalité que de l'écrire mais l'Algérie indépendante aurait gagné à s'inspirer du mode de pensée de Camus. Et dans le rejet persistant vis-à-vis de lui réside, non pas la dénonciation de son ambiguïté par rapport à l'ordre colonial, mais plutôt le refus d'un mode de raisonnement plus ouvert, moins dogmatique, moins définitif. [...] L'unanimisme officiel ne supporte ni réserve ni critique et le questionnement personnel, la nuance dans le propos et l'introspection systématique sont assimilés à de la faiblesse, ou pire, à de la trahison. En fait, nous avons perdu Camus parce qu'il nous est encore interdit de réfléchir comme lui."

\section{Le soleil et le tamis}

7 La pensée qui préside aux destinées des migrants ne s'embarrasse pas de nuances, elle. Puisque crise il y a, il faut empêcher de nouveaux migrants de venir en France. Le volet "contrôle des frontières" prenant le pas sur le volet "intégration" des politiques ministérielles, le bilan 2009 du ministère de l'Immigration n'échappe pas à cette logique. Le 18 janvier, Éric Besson présentant son action se félicitait : "Les flux migratoires continuent d'être mieux maîtrisés", "l'immigration légale comme clandestine régresse. La France reste le premier pays d'Europe en matière d'asile et de naturalisations". 
8 À l'exception des titres de séjour accordés aux étudiants ( $+1,9 \%)$ et aux réfugiés $(+12 \%)$, et bien sûr des "reconduites à la frontière", les statistiques sont en baisse : immigration familiale $(-12,3 \%)$; immigration professionnelle (-15,3\%, 27966 titres de séjour en 2009 contre 33034 en 2008) ; le nombre de titres de séjour long a été de 173 991, soit une baisse de 3,7\% par rapport à 2008 .

29300 reconduites à la frontière (avec une baisse des retours volontaires de 17,9\% et une hausse des retours contraints - 21020 - soit une hausse de 6,5\%). Sur les 47000 demandes d'asile enregistrées, 10864 cartes de séjour au titre de réfugiés ont été accordées (soit un taux d'acceptation de 23,11\%).

10 "Tous les indicateurs disponibles montrent que les flux migratoires, légaux comme illégaux, ont globalement baissé en 2009", a insisté M. Besson, tout en soulignant que cette baisse était "liée, comme dans toute l'Union européenne, à la crise économique mondiale et à la détérioration de la situation de l'emploi". (Le Figaro, 18 janvier). Faudrait-il comprendre que les résultats escomptés viennent moins des rudes politiques mises en œuvre que des caprices de l'économie ? Et puis si les flux baissent, l'immigration, elle, continue. Comme on dit en Kabylie: "Personne ne peut cacher le soleil avec un tamis." Avec 108274 naturalisations, la France, indifférente à ces arguties, continue à intégrer.

11 Autre interprétation, dans Le Monde du 20 janvier : "La crise a surtout un effet sur les flux migratoires économiques au sein des espaces de libre circulation, analyse Jean-Christophe Dumont, de l'Organisation de coopération et de développement économiques (OCDE). [...] L'impact de la crise sur l'immigration économique hors Union européenne reste donc limité. Il semble en revanche plus marqué pour l'immigration familiale." "L'expérience des crises passées montre que le regroupement familial peut être retardé en période de crise, l'étranger présent en France craignant de ne pouvoir assumer le surcoût de la venue de son conjoint ou de sa famille." "Au-delà de la crise, le recul des entrées en France en 2009 s'explique plus, pour M. Dumont, 'par les changements de politiques intervenus ces dernières années que par l'évolution du contexte économique'. Dans le cas de l'immigration familiale, la baisse s'explique également par les mesures des lois sur l'immigration de 2006 et 2007 qui ont durci les conditions de venue des familles (hausse du niveau de ressources autorisant le regroupement familial, allongement du délai pour un Français avant de faire venir son conjoint étranger...)."

\section{Malaises}

De retour d'une tournée qui passait par Marseille, Lyon, Strasbourg, Lille, Cergy, où il a rencontré une centaine d'associations sur le thème des "valeurs de la République", Patrick Gaubert, président du HCI, dresse un premier bilan: "Les inégalités sociales et économiques, les discriminations (particulièrement le contrôle au faciès) sont vécues comme des contradictions fondamentales avec ce qu'ils estiment être des valeurs théoriques de la France républicaine. La multiplication des contrôles de police, leur répétition parfois au cours d'une même journée attise le sentiment de discrimination et aggrave chez les jeunes issus de l'immigration le sentiment d'être des citoyens de seconde zone, voire les renvoie à la condition d'étrangers qu'ont connue leurs parents ou grands-parents." "Quand on demande à ces jeunes s'ils se sentent français, ils vous retournent la question, contestant que le doute ne soit émis que pour eux." (Le Monde, 12 janvier). 
13 Cette situation infligée à au moins deux générations de Français pourrait conduire certains au repli. Ainsi, "plus inquiétant encore, ils ont mis en avant les risques d'un repli identitaire. Ces comportements se justifient, nous ont-ils expliqué, par les difficultés d'intégration, d'insertion dans la société qui entravent le vivre ensemble et par un manque de proximité entre l'État et les citoyens. Lors de certains matchs de football, 'ce n'est pas la France qui est sifflée', selon les associatifs, c'est 'l'État qui ne tient pas parole"”.

14 C'est aussi ce que rapporte Thierry Leclère dans Télérama : "À la tête de la Coordination contre le racisme et l'islamophobie, Abdelaziz Chaambi constate, sur le terrain, les effets collatéraux du débat Besson : 'Le discours sur la citoyenneté ne passe plus auprès des jeunes. Ils ont du mal à se dire français. Les radicaux ont le champ libre et le communautarisme pur et dur, aussi. Sur le plan politique, n'importe quel charmeur de serpent qui avancera au nom de je ne sais quel parti des musulmans peut les récupérer..."' (Télérama n³133, 9 février).

Un repli qui pourrait être accéléré par le fait que "le tissu associatif est en train de mourir, explique P. Gaubert. Il faut le revitaliser, lui donner des moyens. Pour cela il faut examiner la possibilité de passer des conventions avec les centres sociaux, les missions locales, les régies de quartier, et les structures associatives qui, sur le terrain, génèrent du lien social."

16 Si certains s'enferment, dressent des barrières, d'autres, comme les initiateurs de la Journée sans immigrés ( $1^{\mathrm{er}}$ mars), décident de relever la tête et cherchent à changer le regard sur l'immigration. "C'est la mauvaise 'blague' de Brice Hortefeux, visant les Maghrébins sous couvert d'une pique contre les Auvergnats, qui a mis, en septembre 2009, le feu aux poudres. Le débat sur l'identité nationale, sous l'égide du ministère de l'Immigration, n'a pas arrangé les choses. Il y a eu Manuel Valls et ses 'pas assez de Whites', ou Nadine Morano avec sa casquette à l'envers... Je me suis dit: 'Ça suffit, arrêtons de baisser la tête!', explique Karima Ibnou, agent de service hospitalier à Lyon." (Le Monde du 18 février).

“Au début, ces petites grossièretés, si anodines, si ordinaires, les amusaient. Presque. 'Maintenant, ça me gave', décide Karima Ibnou. 'Je voudrais qu'on cesse de me mettre sur le dos tous les maux de la France et de l'Europe - à moi, l'immigré !', renchérit Amadou Gueye, ingénieur en biologie. 'Que les gens réfléchissent avant de nous assigner des places et des rôles qui ne sont pas les nôtres !', ajoute Souhir, étudiante en commerce international."

\section{Les racines du brouillard}

Et pourtant, "93\% des Français estiment vivre dans une société où règne une grande diversité des origines et des cultures et $77 \%$ apprécient cette diversité (27\% la qualifient de très bonne chose et $50 \%$ de bonne chose) contre $17 \%$ qui la qualifient de mauvaise chose", selon un sondage TNS Sofres du 7 février. D'ailleurs, "plus d'un Français sur quatre (28\%) déclare avoir un grand-parent ou un parent d'origine étrangère, et $22 \%$ se disent porteurs de plusieurs cultures. Pour $62 \%$ des sondés, l'intégration est avant tout perçue comme "la possibilité pour un individu de vivre ses différences culturelles, tant que cela ne heurte pas ses concitoyens"'. Schizophrénie ou strabisme divergent? Ou bien la société française serait-elle plus mature que ses dirigeants? 
19 En tout cas, comme d'autres "cherchent les racines du brouillard", il y en a qui tiennent à mesurer cette "diversité". "Ils sont socialistes, écologistes ou membres de la majorité. Et ils se retrouvent sur un point : la mise en place d'une loi sur les 'statistiques de la diversité. Dans un appel rédigé par le Cran (Conseil représentatif des associations noires), Christophe Caresche et Manuel Valls, députés PS, François de Rugy, député Verts, Claude Goasguen et Axel Poniatowski, députés UMP, se prononcent pour des 'statistiques anonymes, autodéclaratives, facultatives et sans constitution de fichiers'." (Le JDD, 5 février). Ils saluent le travail du Comité pour la mesure et l'évaluation de la diversité et des discriminations (Comedd) qui remettait, enfin, le 5 février son rapport à Yazid Sabeg, le commissaire à la Diversité et à l'Égalité des chances, "sur la question très controversée des critères ethno-raciaux". "Au menu, pas de 'statistiques ethniques', évoquées un temps par Nicolas Sarkozy... Mais un panel de divers outils visant à 'calculer la diversité'." (JDD, 6 février 2010).

Et de citer parmi les mesures : "La création d'un observatoire des discriminations rattaché à la Halde et la montée en puissance de la Cnil (Commission nationale de l'informatique et des libertés). Préconisé par Y. Sabeg, le recours au 'ressenti d'appartenance' - à une communauté ne pourra se faire qu'à la marge". "Il ne s'agit pas de compter les communautés pour les contrôler", justifie le démographe François Héran. "Mais il faut que les entreprises, que l'État soient capables de rendre des comptes sur la diversité." "Autre mesure phare préconisée par les 27 experts, l'ajout d'un item portant sur la nationalité et le lieu de naissance des parents dans le recensement de l'Insee. 'C'est le seul moyen de pouvoir suivre la trajectoire des enfants d'immigrés, de voir quelles sont leurs destinées, leurs chances, leur exposition à la discrimination ', assure François Héran."

21 Quelques jours plus tard, Lilian Thuram, Pascal Blanchard, Marc Cheb Sun, Rokhaya Diallo, François Durpaire signaient dans Le Monde du 11 février un appel en faveur d'“ Une République multiculturelle et postraciale". Évoquant les "fissures" d'une "politique de non-mémoire", une "génération postcoloniale" "en quête d'un autre récit historique" et une " fracture coloniale" qui serait "avérée" dans la société, ils demandent à "entrer dans le temps des 'ruptures postcoloniales". “[...] Dans la France du $\mathrm{xxI}^{\mathrm{e}}$ siècle, les 'minorités visibles' restent ignorées du paysage audiovisuel, boycottées à l'Assemblée nationale, absentes du CAC 40, peu nombreuses dans la haute administration ou la diplomatie... Des élites recroquevillées sur elles-mêmes, dans une sorte de République incestueuse. Un État monochrome, comme le sont les médias, pour une nation multicolore. Quel paradoxe! [...] Il faut parler de la 'question raciale' sans la diluer dans la 'question sociale', sans chercher à faire primer l'une sur l'autre, sans vouloir les hiérarchiser au sein de la République. [...] Notre conviction est que la pluralité, partout, doit être encouragée par une action positive à la française."

Pas sûr que cette "action positive à la française" soit du goût de Luc Ferry qui, dans Le Parisien du 10 janvier, dit entendre "beaucoup de gens à droite" lui dire : "On n'a pas voté Sarkozy pour avoir Frédéric Mitterrand, Rama Yade ou Rachida Dati. [...] Si ces gens étaient exceptionnels, pourquoi pas? Mais ils ne sont pas exceptionnels." Et Luc Ferry de poursuivre : "La discrimination positive politique est acceptable. Encore faut-il que ça marche. Fadela Amara, c'est autre chose. Son bilan est ce qu'il est, mais elle représentait avec courage un vrai mouvement, Ni putes ni soumises. Elle se retrouve là grâce au mérite de cette association qu'elle a créée. Les autres, que je sache, n'ont pas brillé par leur utilité." Quid des quotas aux USA ? “ Non, ça a été une catastrophe qui a failli détruire l'université américaine. Le grand exemple de Barack Obama, c'est qu'il n'est pas issu de la discrimination positive. [...] Son parcours est $100 \%$ méritocratique, c'est l'antiquotas. Il n'est pas président par le fait du prince, comme d'autres en France se retrouvent ministres sans avoir rien fait ni avant ni après..." 

création industrielle (ENSCI - Les Ateliers) écrit que "l'ascenseur social ne démarre pas au $15^{e}$ étage !...". Autrement dit, il ne faut pas attendre que les élèves arrivent à la porte des grandes écoles pour commencer à se soucier de l'égalité des chances. Pire et sans circonvolutions diplomatiques : toutes les mesures visant à encourager la promotion de la diversité sont bien gentilles mais ne pourraient être que de la poudre aux yeux si on continue à ignorer les étages scolaires inférieurs. "Les grandes écoles (et les formations universitaires de master), en bout de chaîne, puisent dans les viviers qui leur sont préparés en amont, c'est vers l'amont qu'il faut porter le regard pour essayer d'expliquer (en grande partie) cet élitisme du haut enseignement supérieur."

S'appuyant sur les conclusions de trois études, il montre l'existence d'une chaîne de corrélations entre la présence dans les classes préparatoires aux grandes écoles et le niveau à l'entrée en $6^{\mathrm{e}}$, en CE2 et plus tôt encore... au début du CP ! Ce que Alain Cadix résume par : "Un phénomène de transitivité et d'amplification de l'inégalité scolaire du CP aux CPGE". "Le taux de $30 \%$ de boursiers en CPGE aura un effet mécanique sur la structure sociale des élèves entrant dans les grandes écoles, et cela ira dans le bon sens. Cependant, vouloir imposer un tel pourcentage à toutes, dans les flux entrants, risque de conduire à un déplacement de la sélection. Les grandes écoles [...] vont probablement faire évoluer leurs concours pour permettre une plus grande mixité sociale à l'entrée. Mais il y a fort à parier qu'alors, pour garantir la qualité de leur diplôme principal, [...] certaines grandes écoles seront amenées à créer un diplôme à bac +3 , une licence, comme du reste Sciences Po récemment, transformant la première année d'études en leur sein (troisième et dernière année du cycle licence) en une année de sélection qui sera théorique par ses enseignements et draconienne par ses évaluations. Avec plus de 13 de moyenne (par exemple), l'élève continuera vers le diplôme de la grande école. Entre 10 et 13, il aura la licence et quittera l'établissement. En dessous de 10, il sera réorienté vers l'université. Et nous reviendrons peu ou prou à la case départ tant que le problème ne sera pas pris en charge très en amont."

\section{Tartufes ?}

Claude Weill, sur le Nouvelobs.com (le 26 février), résume: "En l'espace de quelques semaines, nous avons eu droit successivement à l'importation en France du débat helvétohelvétique, inspiré par l'extrême droite suisse, sur la construction des minarets. Puis au grand psy-show national sur la 'burqa' [...]. Ensuite à un magnifique emballement politico-médiatique autour de la candidate NPA du Vaucluse, accompagné d'une ahurissante plainte en justice comme si quelque texte que ce soit, dans l'abondante législation française, pouvait permettre de déchoir de ses droits de citoyenne une femme dont le seul délit est de couvrir ses cheveux d'un voile (en réalité, un foulard noué sur la nuque comme en portaient les femmes de la campagne dans mon enfance).

Et aujourd'hui, c'est le pompon, voilà que le ministre de l'Agriculture dénonce - au nom des principes de la République, bien sûr! - la décision de la chaîne Quick de retirer la viande de porc de quelques établissements."

Et d'éclairer la lanterne de ses lecteurs : "[...] En réalité, ce à quoi on assiste, sous couvert de défense de la République et de lutte contre le 'péril communautariste' (nom de code pour désigner exclusivement le prétendu péril arabo-musulman, car le communautarisme chinois ou juif, tout le monde s'en fout, il est tout à fait accepté et même, à l'occasion, célébré), c'est tout simplement à la mise au ban de l'islam de France. Et ce qui révulse le républicain laïc que je suis, 
c'est que cette sale besogne s'accomplit sous l'invocation des plus nobles idéaux. Ah, les tartufes de la République [...]."

Qu'il y ait des Tartufes cela ne fait aucun doute. Mais il y a aussi de vrais fous furieux. Le 12 janvier, ils ont agressé la comédienne d'origine algérienne Rayhana, qui avait le tort sans doute de ne pas porter de voile, de se soucier de l'existence d'un Quick halal comme de sa première babouche. Qui avait surtout le tort de défendre ses convictions sur une scène de théâtre: "Selon son entourage, Rayhana a été aspergée d'essence et ses agresseurs lui ont ensuite jeté une cigarette au visage, fort heureusement sans enflammer la jeune femme." "L'agression physique s'est doublée d'une agression verbale qui laisse peu de doutes sur le lien existant entre cette tentative d'homicide et les représentations en cours." (Le Monde.fr, 14 janvier).

Oui, il y a des tartufes de la République mais il y a aussi des musulmanes pour qui "vivre ensemble" fait sens. Dans un article sur les "petits arrangements avec le voile", Stéphanie Le Bars rapporte l'exemple de ces femmes qui "se dévoilent à l'entrée de leur entreprise ou de l'établissement public dans lequel elles travaillent. Celles qui étaient lycéennes en 2004, date à laquelle la loi a interdit le port du foulard à l'école, ont vécu avec 'le voile dans le cartable', le retirant à l'entrée de l'établissement. Elles prolongent cette habitude dans le monde professionnel ". (Le Monde, 17 février).

29 Tartufes ou pas, ces femmes méritent autant le respect (et le soutien!) qu'Ilham Moussaïd, qui figurait sur la liste du NPA pour les élections régionales en Paca. " $\mathrm{La}$ réaction des féministes, c'est cela le plus dur, relève la jeune fille, qui a clairement pris position pour la contraception et le droit à l'avortement." Cette candidature provoqua des remous au sein même du NPA. Après un vote, "une 'minorité décide alors de se retirer de la campagne: 'Les convictions religieuses doivent demeurer dans la sphère privée, expliquent les minoritaires [...]. Même si des camarades ont une interprétation progressiste de leur foi, que nous saluons, il n'empêche que les systèmes religieux demeurent de terribles instruments d'oppression'." (Le Monde, 11 février).

N'est-ce pas ce que dit Nadia Weiler-Strasser de l'Association de solidarité avec les femmes arabes qui a déposé un recours en référé pour s'opposer à la présence de la candidate voilée Ilham Moussaïd, sur la liste du NPA ? "En choisissant de porter le voile alors qu'elle prétend à un mandat public, Ilham Moussaïd fait en effet prévaloir ses propres convictions sur les lois républicaines, sur les convictions religieuses et les droits des autres citoyens. Elle porte atteinte à la liberté de conscience de ces derniers et notamment des femmes issues de l'immigration arabo-musulmane qui ne se reconnaissent pas forcément dans les prescriptions imposées par la religion d'Ilham Moussaïd." (Elle.fr, 16 février).

31 Tartufe, René Vandierendonck, le maire socialiste de Roubaix qui a porté plainte contre le Quick de sa ville ? Il "explique ne pas être gêné 'qu'il y ait une offre halal', mais juge que 'ça va trop loin quand on ne propose plus que cela"' (Le Monde.fr, 21 février). Fadela Amara est montée au créneau: "Nous parlons bien ici d'une entreprise privée, qui veut occuper un marché pour faire du business. Je trouve la réaction du maire de Roubaix exagérée et dangereuse. Il n'y a rien de discriminant dans cette histoire : les consommateurs ont le choix de leur menu et peuvent très bien prendre du poisson" (JDD, 21 février). Ce que révèle cette affaire, c'est l'appétit pour un marché halal estimé à plus de cinq milliards d'euros, générateur d'emplois et en pleine croissance.

D'ailleurs, Le Monde du 21 février titrait: "Le Quick halal de Roubaix fait le plein" avant d'ajouter "même si certains clients se sentent "limite un peu choqués". Halima, 21 ans, va au 
Quick “tous les jours depuis qu'il est halal. C'est vrai que les non-musulmans perdent un peu de choix, reconnait-elle, mais si on vend du porc au même comptoir, alors le reste n'est plus halal ". "La population jeune et musulmane de Roubaix interrogée à l'entrée du Quick, vendredi 19 février, exprime un indéniable sentiment de confort face à cette reconnaissance de son identité culturelle et religieuse. Quant aux non-musulmans, nombreux eux aussi ce jour-là dans le fast-food, leurs regrets se manifestent sans animosité. La plupart continuent de fréquenter l'établissement. Faute d'alternative aussi."

Pendant ce temps, il en est qui décident d'aller voir si l'herbe des pays dits musulmans y est plus verte. "Rêve d'islam" montre ces Français qui partent vivre leur foi en "pays musulman" ("hijrah"). Ils se prénomment Moussa, Meriem, deux convertis, Khaled ou Abdellak (Le Monde 20 janvier).

Pour l'imam de Bordeaux, Tareq Oubrou, "les jeunes des nouvelles générations envisagent la hijrah pour trouver du travail et pratiquer l'islam de manière visible tout en passant inaperçus. Certains vivent en effet leur religiosité en France avec douleur à cause du climat médiatique et sociétal." "Pratiquer l'islam de manière visible" ? Serait-ce un euphémisme pour évoquer le statut de la femme, les libertés individuelles, es libertés démocratiques et pour n'avoir le droit de pratiquer qu'un islam unique, univoque et unanimiste? D'ailleurs, pour Moussa, converti à la "barbe longue et fournie", pour qui "la France et l'islam sont incompatibles", "[...] l'islam est unique et il n'y a pas un islam plus 'rigoriste' 'qu'un autre". Et de dénoncer "l'islam 'du juste milieu' qui, en Europe, autorise les musulmans à adapter une partie de leurs pratiques au contexte socioculturel dans lequel ils vivent."

Mais voilà, selon M. Oubrou, "les expatriés rentrent au bout de quelques années. Ils se rendent compte que la France est un pays libre avec des avantages sociaux non négligeables! [...] Ces exils avortés sont connus de la communauté musulmane, mais rares sont ceux qui acceptent d'en parler." De plus, "les gouvernements des pays concernés ne voient pas toujours d'un bon ceil l'arrivée de musulmans pieux, susceptibles de verser dans le radicalisme ou l'islamisme. 'Dans la banlieue du Caire, des Français musulmans sont montrés du doigt par les Égyptiens qui les trouvent trop radicaux dans leurs pratiques', explique le chercheur Franck Frégosi. Une suspicion que l'on observe aussi de la part des gouvernements dans les 'pays d'origine'." Que disait Camus déjà ? Notre tâche est de combattre "nos bagnes et nos ravages", "en nousmêmes et dans les autres"... 